\title{
Prevalencia, características clínicas y socioeconómicas de teniasis y cisticercosis en una población de Lurín - 2004
}

\author{
Prevalence, clinical and socio-economic characteristics of teniasis \\ and cysticercosis in a population of Lurin - 2004
}

\author{
Ofelia Castillo-Contreras ${ }^{1}$, Juan De Coll-Vela ${ }^{2}$, Jorge Lermo-Sandoval ${ }^{3}$, \\ Gregory López-Peña ${ }^{4}$, Julián Vega-Peláez
}

Resumen

Objetivo: Determinar la prevalencia de teniasis intestinal y seroprevalencia de cisticercosis de los vendedores de chicharrones de Lurín en Lima-Perú, y conocer sus características epidemiológicas, socioeconómicas y síntomas asociados con la parasitosis. Material y métodos: Se realizó un estudio transversal en vendedores de chicharrones del asentamiento humano "Julio C. Tello" de Lurín. Previo consentimiento informado de los participantes. Se utilizó una encuesta socioepidemiológica y se recolectó muestras de sangre para detectar anticuerpos específicos a Cisticerco (EITB) y de heces para la detección de coproantigenos de Taenia sp. (ELISA). Las muestras se procesaron en el Laboratorio de Cisticercosis del Instituto Nacional de Ciencias Neurológicas. El protocolo fue aprobado por el Comité de Ética de la Facultad de Medicina de San Fernando. Resultados: De 49 encuestados, el 89,8\% participaba en la preparación de chicharrones y atención al público. El 81,6\% refirió lavarse las manos siempre después de defecar, $65,3 \%$ refirió hacerlo antes de consumir alimentos. Hubo 41 muestras de sangre que resultaron negativas para cisticercosis. La detección de coproantigenos de Taenia sp. fueron negativos en todos los casos. No se encontró huevos de Taenia sp. en el examen de microsedimentación. Los locales contaban con servicios de agua y desagüe. Discusión: Las condiciones sanitarias y hábitos higiénicos adecuados reducirían el riesgo de infección. El consumo de carne de cerdo en forma de chicharrón podría no estar asociado a la trasmisión de este parásito. Conclusión: Los datos obtenidos indican que no existe teniasis ni cisticercosis en dicha población estudiada.

Palabras clave: Chicharrones, cisticercosis, neurocisticercosis, Perú, teniasis.

\section{Abstract}

Objectve: To determine the prevalence of intestinal taeniasis and seroprevalence of cysticercosis of the fried pork seller from Lurin in Lima-Perú and to know the epidemiological, socio-economic characteristics and asociated symptoms with the parasitosis. Material and methods: We carried out a tranversal descriptive study with the fried pork seller from an urban community "Julio C. Tello" in Lurin. We get a informed assent of everybody participants. We carried out a socioepidemiological survey and we took samples of blood to detect specific antibodies to cysticerci (EITB) and of feces for the detection of coproantigenos of Taenia $s p$. (ELISA). All the samples were analyzed in Cysticercosis's Laboratory of the Institute National of Neurological Sciences. The protocol was approved by the Committee of Ethics of the Faculty of San Fernando's Medicine Results: Of 49 surveyed, the $89.8 \%$ of the population was participating in the preparation of fried pork and attention to the public. $81.6 \%$ support that they always wash their hands after defecating, and $65.3 \%$ support that they always do it before consuming food. There were 41 blood samples that were negative for cysticercosis. The detection of coproantigens of Taenia sp. they were negative in all cases. There were no eggs of Taenia $s p$. in the examination of microsedimentation. The places for selling the fried pork have basic services. Discussion: The sanitary conditions and hygienic suitable habits would reduce the risk of infection. The consumption of meat of pig in the shape of fried pork might not be associated with the transmission of this parasite. Conclusion: The obtained information indicates that there's no taeniasis and cysticercosis in the studied population.

Keywords: Cysticercosis, fried pork, neurocysticercosis, Perú, taeniasis.

\footnotetext{
${ }^{1}$ Médico gastroenteróloga del Hospital Nacional Edgardo Rebagliati Martins, EsSalud. Lima-Perú. ORCID: htpp//orcid.org/00000003-3017-0169. ${ }^{2}$ Medicina Familiar y Comunitaria. Hospital Príncipe de Asturias, Madrid- España. ORCID: http://orcid.org/00000002-0776-448X. 3Jorge Lermo-Sandoval: Urología. Hospital Universitario Quironsalud Madrid, Madrid-España. ORCID: http://orcid.org/0000-0003-3032-3444. ${ }^{4}$ Gregory López-Peña: Medicina Física y Rehabilitación. Instituto Nacional de Rehabilitación, Lima-Perú. ORCID: http://orcid.org/0000-0003-3191-6786. 'Julián Vega-Peláez: Médico cirujano. Diplomatura en auditoría médica . ORCID: https://orcid.org/0000-0002-9734-9123.
} 


\section{Introducción}

La teniasis por Taenia solium y cisticercosis son enfermedades infecciosas endémicas en los países en vías de desarrollo $^{(1)}$ y aún en países industrializados ha aumentado la incidencia por la llegada de inmigrantes de zonas endémicas ${ }^{(2)}$. Los seres humanos, hospederos definitivos del estadio adulto, desarrollan teniasis después de comer carne de cerdo poco cocida o cruda que contenga la forma larval del parásito. Tanto las personas como los cerdos pueden desarrollar cisticercosis al ingerir huevos procedentes de un portador de Taenia solium. La cisticercosis se establece en diferentes tejidos pero sobrevive por mayor tiempo en el sistema nervioso o el globo ocular, lugares importantes de morbilidad de cisticercosis ${ }^{(3)}$. Las convulsiones son la forma de presentación más común en $50 \%$ $70 \%$ de los casos de neurocisticercosis. Ésta se adquiere en una variedad de condiciones culturales y socioeconómicas en las que exista un contacto cercano con un portador de Taenia solium a través del agua, manos o comida contaminada con huevos $^{(4)}$; se ha encontrado que entre un $15 \%-25 \%$ de casos también tienen teniasis lo que sugeriría autoinfección ${ }^{(5)}$. En la mayoría de países en vías de desarrollo el ciclo vital de este parásito se mantiene por las deficientes condiciones sanitarias y crianza doméstica de cerdos sin control veterinario y sin vigilancia por las autoridades respectivas ${ }^{(6)}$.

La observación microscópica de huevos o proglótides de Taenia solium en heces, muchas veces lleva a error diagnóstico debido a la bajas sensibilidad y especificidad ${ }^{(7-8)}$, además que morfológicamente son indistinguibles de los huevos de Taenia saginata. Se ha desarrollado un test de ELISA que detecta copro-antígenos con una sensibilidad mayor a $90 \%$ y especificidad de $99,2 \%$, que ha demostrado un incremento en la detección de casos de Taenia solium de por lo menos 2,6 veces más comparado con microscopía ${ }^{(9-10)}$.

Se han publicado varias técnicas de biología molecular que permiten un diagnóstico de especie, como la Reacción en Cadena de Polimerasa. Una de las pruebas más específicas para el diagnóstico de cisticercosis es la técnica de Inmunoelectrotransferencia (EITB) o "Western Blot", un inmunoblot de 7 glucoproteínas cuya especificidad es del $100 \%$ y una sensibilidad del $98 \%{ }^{(11)}$. La detección de estos anticuerpos específicos a cisticerco indica exposición al parásito y no necesariamente una infección establecida debido a los anticuerpos transitorios $^{(11)}$. Sin embargo, estos métodos son aplicados para estimar la prevalencia e identificar factores de riesgo asociados a la infección de Taenia solium ${ }^{(11-13)}$.

En el Perú se han llevado a cabo estudios de seroprevalencia de cisticercosis en sus tres regiones: costa, sierra y selva, comunicándose una alta prevalencia de teniasis y cisticercosis humana y en cerdos en la sierra central relacionados con la crianza de cerdos para el consumo y venta de carne ${ }^{(13,14)}$, sin control sanitario $^{(6)}$. En Lurín, provincia de Lima; la crianza de cerdos, tanto en granjas como en domicilios, son costumbres tradicionales; así como la venta de chicharrones, sobre todo los sábados y domingos, en especial en el AAHH "Julio C. Tello", en el lugar conocido como "Puente Lurín"; ubicado en la Antigua Panamericana Sur.

La carencia de estudios previos sobre teniasis y cisticercosis en dicha zona, así como la alta concurrencia a los establecimientos por los visitantes, representan razones para descartar un probable foco de transmisión de la enfermedad. El objetivo general de esta investigación fue determinar la prevalencia de teniasis intestinal y seroprevalencia de cisticercosis en los vendedores de "chicharrones" del AAHH "Julio C. Tello" - Lurín. Los objetivos específicos fueron conocer las características demográficas, socioeconómicas y síntomas asociados a esta parasitosis.

\section{Material y métodos}

El presente estudio fue de tipo observacional de corte transversal, se realizó en el A.A.H.H "Julio C. Tello" en el extremo norte del distrito de Lurín entre el Km. 23,7 y Km. 29 de la autopista Panamericana Sur, provincia de Lima - Perú.

Se realizó un análisis situacional del AAHH "Julio C. Tello" con el objetivo de identificar los locales en donde se expenden chicharrones, los que fueron censados para determinar el número de personas que intervienen en el proceso de venta, conformando una población de 74 individuos. Se contactó a los dirigentes de los vendedores de chicharrones con el objetivo de convocar a una reunión general de toda la población de vendedores, donde se informó acerca del ciclo vital y de los riesgos de teniasis y cisticercosis. Se les explicó el consentimiento informado a las personas que deseaban participar en el estudio, donde se les ofrecía diagnóstico y tratamiento totalmente gratuito a los que tendrían resultado positivo.

Se realizó el estudio en la población elegida tomándose como criterios de inclusión: Ser vendedores de chicharrones (personas que de manera directa intervienen en la preparación y atención en los establecimientos donde se expenden chicharrones con más de 24 horas por semana y por lo menos durante un mes, así como las personas que lo hacen indirectamente por residir en el mismo local), acceder a participar en el estudio previa aceptación del consentimiento informado. Como criterio de exclusión: No aceptar participar en el estudio y/o no firmar el consentimiento informado.

A las personas enroladas se les visitó en su local y se recolectó información por medio de una encuesta en formatos diseñados para este fin, que incluyó variables demográficas (nombre, sexo, edad), socioeconómicas (número de habitantes por vivienda, número de habitaciones, crianza de cerdos, abastecimiento de agua y desagüe) y clínicas (historia de convulsiones, mareos, cefalea e historia de haber expulsado proglótides de tenia con las deposiciones). Al mismo tiempo se les tomó, previa desinfección con alcohol, una muestra de sangre ( 2 a $5 \mathrm{ml}$ de sangre con tubo de ensayo con presión negativa) para detectar anticuerpos específicos a Cisticerco por el método EITB (Western Blot para Cisticercosis). Además se brindó envases plásticos para la colección de las muestras de heces las que fueron destinadas a la detección de coproantígenos de Taenia $s p$. por el método ELISA. Todas las muestras, tanto de sangre como de heces se procesaron en el Laboratorio de Cisticercosis del Instituto Nacional de Ciencias Neurológicas en Lima, y debido a la especificidad de género en el test de ELISA, todas las muestras positivas a coproantígenos de Taenia $s p$. fueron analizadas por la técnica de Reacción en Cadena de Polimerasa a cargo de The Cisticercosis Working Group in Peru - siglas CDC, para conocer los casos reales de Taenia solium y excluir los casos de Taenia saginata. 
Para el presente estudio se definió seropositivo como un individuo que es sometido a un examen serológico para detectar anticuerpos a Cisticerco, mediante el método EITB (Western Blot) y cuyo resultado revela presencia de por lo menos una banda reactiva. Se definió como portador de Taenia $s p$ a un individuo que es sometido a descarte de teniasis intestinal por detección de antígenos de Taenia sp. en heces y cuyo resultado indica positividad cuando hay una reacción colorimétrica (método ELISA).

Se definió portador de Taenia solium al individuo con muestra positivo para copro-antígenos de Taenia sp. Y resultado positivo para Taenia solium por la técnica de Reacción en Cadena de Polimerasa.

El análisis de los datos se realizó mediante estadística descriptiva, usando la presentación de distribución de frecuencias (Tablas y Figuras). El procesamiento electrónico se realizó en el paquete SPSS versión 11.0.

\section{Aspectos éticos}

El protocolo fue aprobado por el Comité de Ética de la Facultad de Medicina de San Fernando, Universidad Nacional Mayor de San Marcos y presentado a la Asociación Gremial de Chicharroneros de la zona. Todos los participantes en el estudio firmaron un consentimiento informado, donde se explica el propósito del estudio, los procedimientos de los análisis, los beneficios de la gratuidad del diagnóstico y tratamiento en los casos positivos, así como sus derechos de autonomía y decisión.

\section{Resultados}

La población de vendedores de chicharrones censada incluyó un total de 74 habitantes mayores de edad, 30 varones y 44 mujeres, correspondientes a 19 chicharronerías. Cuarenta y nueve $(66,2 \%)$ fueron 19 varones y 30 mujeres (Tabla 1$) ; 41$ $(83,7 \%)$ encuestados proporcionaron muestras de sangre y 32 $(43,2 \%)$ de ellos también muestras de heces (Tabla 2).

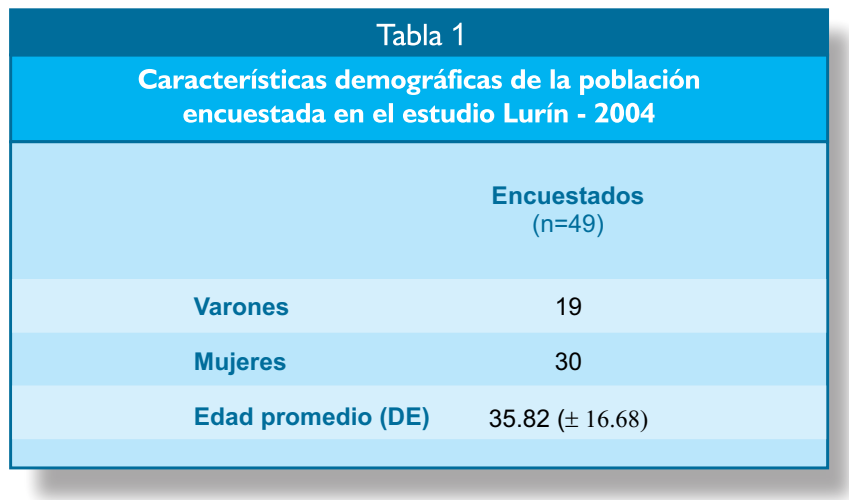

Fuente: La Investigación.

Cuarenta y cuatro entrevistados $(89,8 \%)$ participaron directamente en la preparación de chicharrones y atención al público; 48 (98\%) señalaron consumir carne de cerdo, de ellos

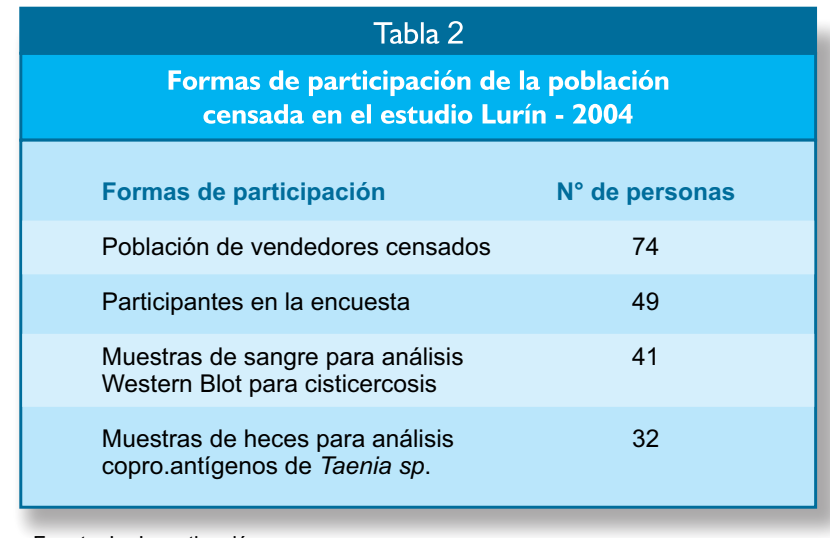

Fuente: La Investigación.

la mayoría admitió consumirlo semanalmente $42(85,7 \%)$ y 6 $(12,2 \%)$ vendedores diariamente.

Las $41(83,6 \%)$ muestras de sangres analizadas con EITB resultaron negativos para cisticercosis. La detección de copro-antígenos de Taenia sp. por el método ELISA resultaron negativos en todos los casos y en el examen de microsedimentación no se encontró huevos de Taenia sp. sin embargo se halló quistes y huevos de otros parásitos (Tabla 3).

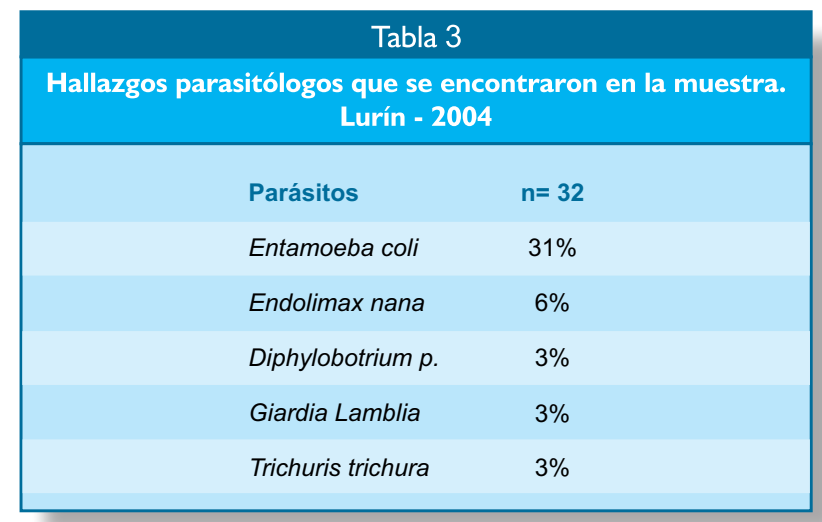

Fuente: La Investigación.

Todos los locales son de material noble y cuentan durante las 24 horas del día con servicios de agua potable, desagüe, luz eléctrica. La mayoría de los locales adquieren la carne de cerdo de la granja $(61,2 \%)$ y mercado $(10,2 \%)$; y 35 $(71,4 \%)$ afirman que la carne cuenta con control sanitario. Cuarenta y siete $(95,9 \%)$ de los encuestados no cría cerdos actualmente. Respecto a los hábitos higiénicos, 40 (81,6\%) refirió lavarse las manos siempre después de defecar y 32 $(65,3 \%)$ siempre antes de consumir alimentos y bebidas.

De acuerdo con los síntomas neurológicos que alguna vez habían presentado los encuestados mencionaron: 18 $(36,7 \%)$ dolores de cabeza 12 refirieron más de 1 vez por semana, $13(26,5 \%)$ mareos, y solo $2(4,1 \%)$ refirieron sufrir de "ataques" (Figura 1); de ellos una mujer de 20 años presentó antecedente de convulsiones el año anterior y el EITB y coproantígeno en heces resultaron negativos, el 


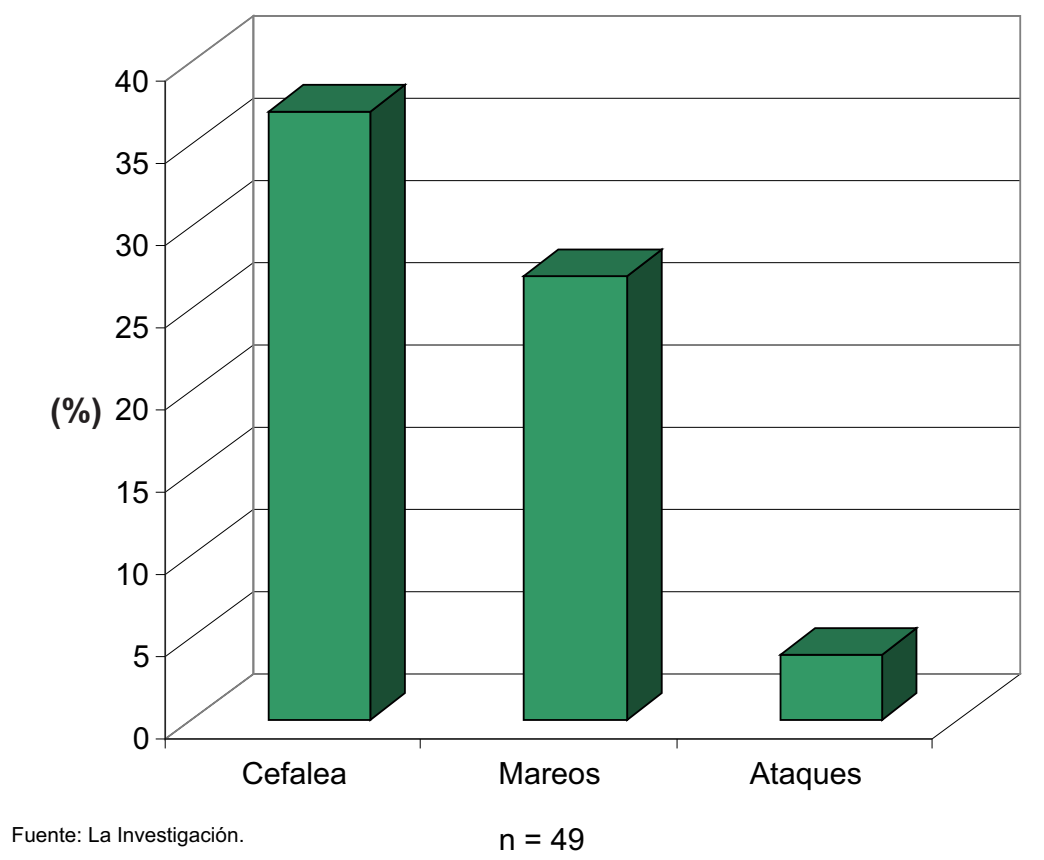

Figura 1. Presencia d síntomas neurológicas que alguna vez habían presentado en el último año. Lurín - 2004

segundo un varón de 43 años tuvo convulsiones hace 10 años pero se negó a la toma de muestras.

Solo $4(8,2 \%)$ entrevistados reconocieron haber eliminado alguna vez gusanos largos y planos, y 5 (10,2\%) refirieron tener familiares que también eliminaron gusanos. Ninguno refirió diagnóstico de cisticercosis ni antecedentes familiares de dicha enfermedad. El 24 (49\%) desconocían la relación de la enfermedad con el consumo de carne de cerdo y la forma de contagio (Figura 2).

\section{Discusión}

La Teniasis y Cisticercosis son altamente prevalentes en Perú, donde elevadas tasas de infección por T. Solium han sido comunicados tanto en humanos como en $\operatorname{cerdos}^{(6,15)}$ Estudios realizados por García et al., en diferentes regiones del Perú muestran una alta prevalencia de infección en humanos por este parásito en comunidades de la sierra $(13 \%$ en Haparquilla y $24 \%$ en Saylla) y de la Selva (Maceda $8 \%$ y Churusapa 17\%).

En el estudio se halló una seroprevalencia nula de cisticercosis y ningún caso de infección por Taenia Solium. Estos resultados difieren de lo reportado por García et al., en vendedores de chicharrones de la comunidad andina de Saylla, cerca a Cusco, en donde se encontró una seroprevalencia de cisticercosis de $23,3 \%$ y una prevalencia de teniasis de $8,6 \%{ }^{(14)}$ Las diferencias podrían deberse a las menores condiciones sanitarias en la comunidad de Saylla tales como un menor acceso a desagüe, y sobretodo a la crianza doméstica de cerdos, por parte de los pobladores, posiblemente en malas condiciones; mientras que en nuestra población todos los "chicharroneros" estudiados refirieron tener en sus locales servicio de desagüe y agua potable, la procedencia de la carne de cerdo para la venta tenia control sanitario y en su mayoría provenía de granjas y mercados, y solo 2 personas encuestadas aseveraron criar cerdos.

Otro estudio realizado en la comunidad costeña de Monteredondo, en el departamento de Piura, encontró también una alta seroprevalencia a cisticercosis en humanos $(16 \%)^{(16)}$. Sin embargo estos estudios fueron realizados en lugares del Perú señalados como endémicos. En zonas no endémicas se reporta baja prevalencia de teniasis y cisticercosis, tal como lo demuestra el estudio efectuado en grupos urbanos no seleccionados de Lima, el cual halló una seroprevalencia de alrededor de $1 \%$ o menos ${ }^{(17)}$. Un estudio en Lagamar, Brasil no encontró asociación estadística entre el hábito de consumir carne de cerdo y la infección por Taenia solium ${ }^{(18)}$. Así mismo, resultados hallados por García et al., acerca del consumo de carne de cerdo en la forma de "chicharrón", sostienen que las características de cocción a alta temperatura y por largo tiempo, hacen improbable la sobrevivencia del cisticerco y por lo tanto la transmisión de la teniasis ${ }^{(14)}$. Según estos estudios, al tener nuestra población la costumbre de consumir chicharrones, haría poco probable la transmisión de teniasis, esto se correlaciona con nuestros resultados negativos a coproantigenos de Taenia sp. en todos los casos. Otro punto importante a considerar es que las personas dedicadas a criar de manera doméstica cerdos tienen alta probabilidad de presentar anticuerpos a Taenia solium; y esta probabilidad se incrementa si además el comerciante mata los cerdos en su casa ${ }^{(14)}$. Estas costumbres no fueron referidas por las personas encuestadas en el estudio. 


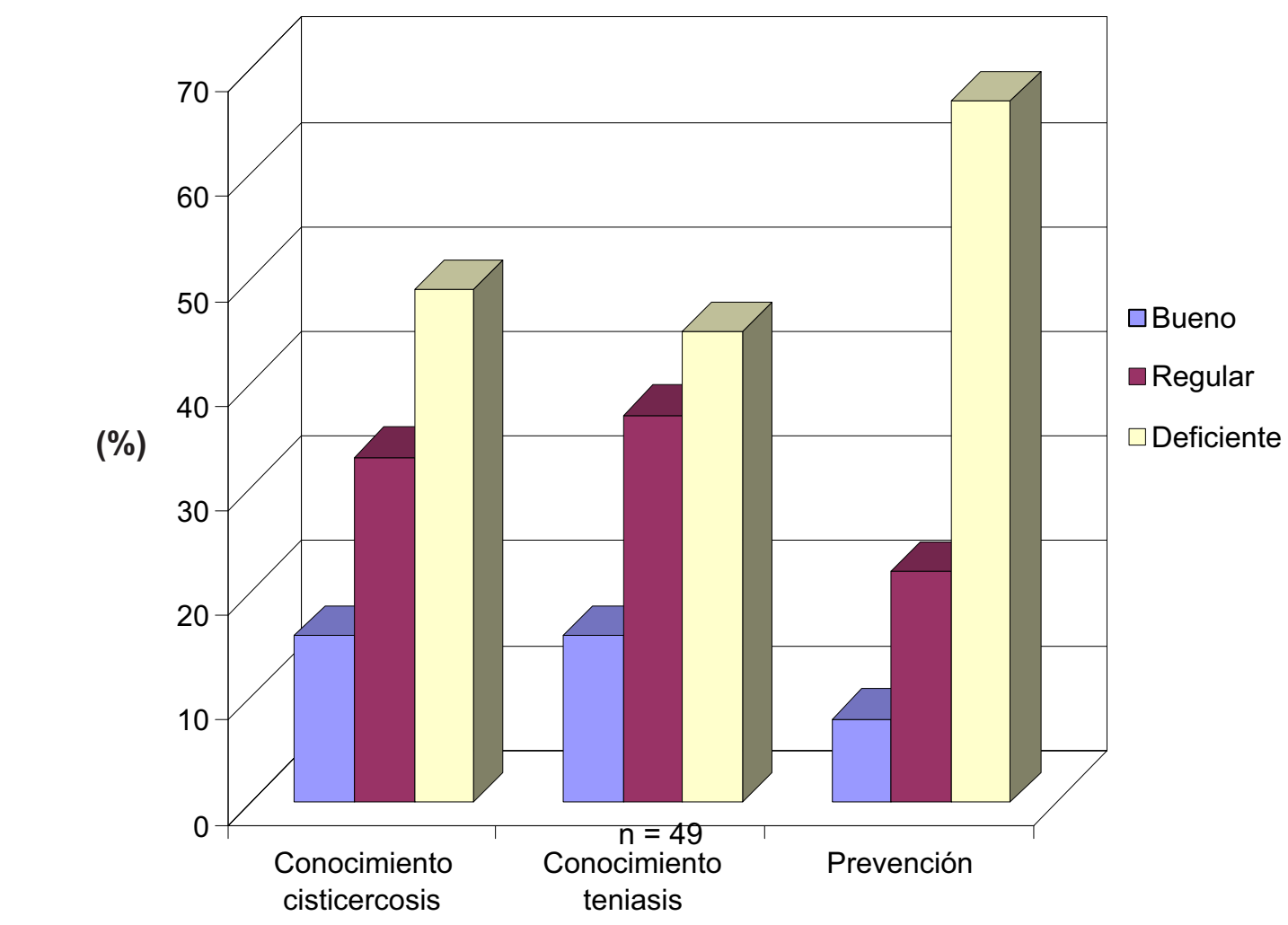

Fuente: La Investigación.

Figura 2. Conocimientos sobre Teniasis y Cisticercosis de la población. Lurín - 2004

Entre las limitaciones tenemos el tamaño de la muestra debido a la participación parcial de los vendedores de chicharrones. El 33\% del total de vendedores del AAHH "Julio C. Tello" no participó, esto debido a la desconfianza de los vendedores, manifestado en el censo poblacional, sobretodo por el manejo de sus resultados por parte de los investigadores temiendo el perjuicio de sus establecimientos, así mismo el miedo a la punción venosa y el desinterés de entregar las muestras de heces para el estudio.

Un estudio realizado en un área sub-urbana de la ciudad de México en una población de comerciantes de alimentos preparados con carne de cerdo encontraron solo un caso positivo a cisticercosis y ningún portador de Taenia solium ${ }^{(19)}$. Dicha población tenía establecimientos con servicios de agua potable y desagüe, además refirieron tener adecuados hábitos higiénicos y que la carne de cerdo para la venta provenía en su mayoría de mercados. El único caso positivo a cisticercosis representó al $0,9 \%$ de seroprevalencia. Teniendo esta población características semejantes a la nuestra, se podría estimar una baja seroprevalencia de cisticercosis y teniasis en nuestro estudio. Las condiciones sanitarias y hábitos higiénicos adecuados reducirían el riesgo de infección.

\section{Conclusiones}

En el presente trabajo se concluye que existe una prevalencia nula de teniasis y cisticercosis en la población estudiada. La cefalea y los mareos son los síntomas neurológicos más frecuentes que presentaron como antecedente.

El consumo de carne de cerdo en forma de chicharrón podría no estar asociado a la transmisión de este parásito. Debido a la intervención de varios factores en la transmisión de esta parasitosis sería apropiado incluir en futuras investigaciones a los cerdos de los camales, tanto formales como clandestinos del distrito de Lurín, por representar la cisticercosis porcina un buen indicador epidemiológico de la infección en humanos; así como reproducir el estudio en otras poblaciones; no necesariamente relacionadas con la venta $y / 0$ consumo de carne de cerdo.

\section{Agradecimientos}

Expresamos nuestro especial agradecimiento por su apoyo invalorable al Dr. Hugo García, Jefe del Laboratorio de Cisticercosis del Instituto Nacional de Ciencias Neurológicas, a la Licenciada en Biología Silvia Rodríguez, a la Licenciada en Enfermería Sandra Choqueneira, así como a los estudiantes de la Universidad Nacional Mayor de San Marcos (UNMSM) Álvaro Arroyo Salgado, Jenny Gómez Delgado y Karla Salvatierra Lucano, cuya colaboración permitió la realización de este trabajo. En especial al Dr. Luis Torres Ramírez, del Instituto Nacional de Ciencias Neurológicas por su apoyo en el manuscrito del artículo. 
1.García HH, Gonzáles A, Evans C, Gilman RH. The Cysticercosis Working Group in Peru. Taenia solium cysticercosis. Lancet 2003;362:547-556.

2.Schantz PM, Moore AC, Munoz JL, et al. Neurocysticercosis in an Orthodox Jewish community in New York City. N Engl J Med 1992;327;692-695.

3.Garcia HH, Gonzales AE. Teniasis por Taenia solium. Rev. Diagnóstico. 2000;39(4):176-178.

4.Gilman RH, García HH, Gonzáles AE, et al. Short cuts to development: methods to control the transmission of cysticercosis in developing countries. In: García HH, Martínez SM, eds. Taenia solium taeniasis/ cysticercosis, 2d Ed. Lima: Ed Universo, 1999;313-326.

5. Gilman RH, Del Brutto OH, García HH, et al. Prevalence of taeniosis among patients with neurocysticercosis is related to severity of infection. The Cysticercosis Working Group in Peru. Neurology 2000;55:1062.

6. The Cysticercosis Working Group in Peru. The marketing of cysticercotic pigs in the Sierra of Peru. The Cysticercosis Working Group in Peru. Bull World Health Organ 1993;71:223228.

7. Allan James C. Detection of Taenia solium Antigens in feces. In: García HH, Martínez SM, eds. Taenia solium taeniasis/ cysticercosis, 2d Ed. Lima: Ed Universo, 1999;59-67.

8. Allan JC, Wilkins PP, Tsang VC, et al. Inmunodiagnostic tools for taeniasis. Acta Trop. 2003;87:87-93.

9. Allan JC, Velásquez-Tohom M, Garcia-Noval J, et al. Field trial of the coproantigen-based diagnostic of Taenia solium taeniasis by enzyme-linked immunosorbent assay. Am. J. Trop. Med. Hyg. 1996;54:352-356.

10. Allan JC, Craig PS, García-Noval J. Coproantigen detection for immunodiagnosis of echinococcosis and taeniasis in dogs and humans. Parasitology 1992;104:347-356.
11. Dorny P, Brandt J, Zoli A, et al. Inmunodiagnostic tools for human and porcine cysticercosis. Acta Trop. 2003;87:79-86.

12. Tsang VC, Garcia HH. Immunoblot diagnostic test (EITB) for Taenia solium Cysticercosis and its contribution to the definition of the under-recognized but serious Public Health Problem. In: García HH, Martínez SM, eds. Taenia solium taeniasis/ cysticercosis, 2d Ed. Lima: Ed Universo, 1999.245252.

13. Garcia HH, Del Brutto O. Taenia solium cysticercosis. Infect Dis Clin North Am 2000;14:97-119.

14. García HH, Araoz R, Gilman RH, et al. Increased prevalence for cysticercosis and taeniasis among professional fried pork vendors and the general population of a village in the Peruvian highlands. The Cisticercosis Working Group in Peru. Am J Trop Med Hyg 1998; 59: 902-905.

15. Diaz F, García HH, Gilman RH, et al. Epidemiology of taeniasis and cysticercosis in a Peruvian village. The Cisticercosis Working Group in Peru. Am J Epidemiology 1992; 135:875-882.

16.García HH, Gilman RH, González AE and The Cisticercosis Working Group in Peru. Epidemiology of Taenia solium infection in Peru. In: García HH, Martínez SM, eds. Taenia solium taeniasis/ cysticercosis, 2d Ed. Lima: Ed Universo, 1999;297-305.

17. García HH, Martínez SM, Gilman RH, et al. Diagnosis of cysticercosis in endemic regions. The Cysticercosis Working Group in Peru. Lancet 1991;338:549-551.

18. León SV, Prata A, Silveira N. Risk factors associated with taeniasis-cysticercosis in Lagamar, Minas Gerais State, Brazil. Rev Soc Bras de Med Trop. 1998;31(1):65-71.

19. Cruz V, Plancarte A, Morán I, et al. Teniosis y cisticercosis en comerciantes de alimentos en mercados de una área de la ciudad de México. Parasitol Latinoam 2003;58:41-48.

Contribución de autoría: Ofelia Castillo-Contreras participó en la recolección de datos, diseño del artículo, revisión crítica del artículo y en la aprobación de la versión final. Juan De Coll-Vela participó en la recolección de datos, diseño del artículo y en la aprobación final. Jorge Lermo-Sandoval participó la recolección de datos, y en la aprobación de la versión final. Gregory López-Peña participó en la recolección de datos, redacción del manuscrito y en la aprobación

final. Julián Vega-Peláez participó en la recolección de datos, redacción del manuscrito y en la aprobación final.

Conflicto de interés: Los autores no tienen conflictos de interés con la publicación de este trabajo.

Financiamiento: Autofinanciado.

Citar como: Castillo-Contreras O., y cols. Prevalencia, características clínicas y socioeconómicas de teniasis y

cisticercosis en una población de Lurín - 2004. Diagnóstico(Lima). 2020;59(4):173-178.

DOI:10.33734/diagnostico.v59i4.251

Correspondencia: Ofelia Castillo Contreras. Correo electrónico: brisaida2@yahoo.es

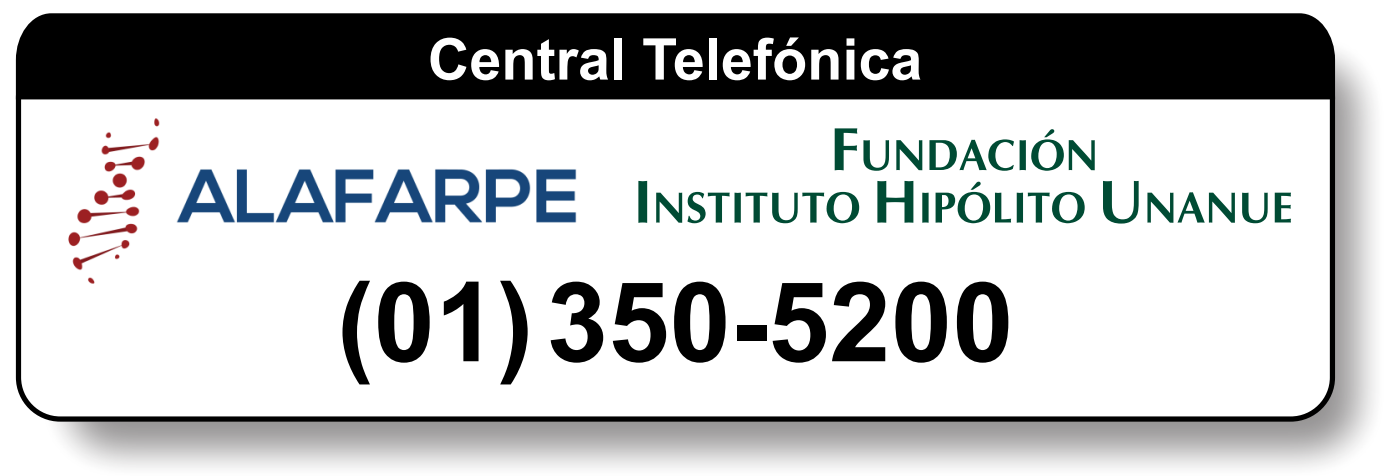

